\title{
The role of molecular imaging in the evaluation of myocardial and peripheral angiogenesis
}

\author{
Mitchel R. Stacy $•$ Jin Chul Paeng • \\ Albert J. Sinusas
}

Received: 20 February 2015/ Accepted: 26 February 2015/Published online: 8 March 2015

(C) The Japanese Society of Nuclear Medicine 2015

\begin{abstract}
Angiogenesis, or the formation of new microvasculature, is a physiological process that may occur in the setting of chronic tissue ischemia and can play an important role in improving tissue perfusion and blood flow following myocardial infarction or in the presence of peripheral vascular disease (PVD). Molecular imaging of angiogenesis within the cardiovascular system is a developing field of study. Targeted imaging of angiogenesis has the potential for non-invasive assessment of the underlying molecular signaling events associated with the angiogenic process and, when applied in conjunction with physiological perfusion imaging, may be utilized to predict and evaluate clinical outcomes in the setting of ischemic heart disease or PVD. This review discusses the developing radiotracer-based imaging techniques and technology currently in use that possess potential for clinical translation, with specific focus on PET and SPECT imaging of myocardial and peripheral angiogenesis.
\end{abstract}

Keywords Myocardial angiogenesis - Peripheral angiogenesis $\cdot$ Molecular imaging $\cdot$ PET $\cdot$ SPECT

M. R. Stacy · A. J. Sinusas

Department of Internal Medicine, Section of Cardiovascular

Medicine, Yale University School of Medicine,

Dana-3, P.O. Box 208017, New Haven, CT 06520, USA

J. C. Paeng

Department of Nuclear Medicine, Seoul National University Hospital, Seoul, South Korea

A. J. Sinusas $(\bowtie)$

Department of Diagnostic Radiology, Yale University School of Medicine, New Haven, CT 06520, USA

e-mail: albert.sinusas@yale.edu

\section{Introduction}

Molecular imaging is a technique that is focused on the non-invasive assessment of in vivo molecular and associated physiological processes. The most established modalities used for molecular imaging are single photon emission computed tomography (SPECT) and positron emission tomography (PET), which use radiotracers that are capable of targeting specific biological events associated with disease progression and therapeutic success. Both SPECT and PET possess high sensitivity and good tissue penetration depth, making them ideal modalities for targeted molecular imaging. The development of hybrid imaging systems in recent years now permits co-localization of high-sensitivity functional SPECT and PET images with high-spatial resolution anatomical images from X-ray computed tomography (CT) and magnetic resonance (MR) systems. These hybrid systems allow for attenuation correction and partial volume correction, thereby improving localization and quantification of radiotracer uptake within specific anatomical regions of interest (e.g., organ systems, soft tissue, vascular networks) [1].

The most established modality for molecular imaging is PET; however, SPECT imaging offers some advantages. SPECT cameras are less expensive and more widely available compared to PET systems. SPECT systems allow for the routine use of dual radioisotope imaging through the differentiation of gamma emitters, thus permitting simultaneous imaging of molecular and physiological indices, such as angiogenesis and tissue perfusion. The use of PET systems continues to grow due to increased availability of hybrid systems with higher sensitivity for detection of molecular events and higher spatial resolution imaging than traditional SPECT systems. Additionally, PET imaging commonly incorporates radiotracers with shorter half- 
lives, which ultimately results in decreased patient exposure to ionizing radiation [2]. Recent pre-clinical work suggests the possibility of dual-isotope PET imaging using pure positron emitters (e.g., nitrogen-13, fluorine-18) and radiotracers that emit a prompt gamma (e.g., rubidium-82, bromine-76, iodine-124) in association with the primary beta, allowing newer PET systems with faster electronics and shorter detector decay constants the ability to detect coincidences and triplets [3, 4].

One physiological process of significant interest in the medical community that may be better evaluated through the use of radiotracer-based molecular imaging is angiogenesis, a process that plays an important role in the restoration of tissue perfusion and blood flow following the onset of myocardial and skeletal muscle ischemia [5]. Traditional assessment of angiogenesis has been restricted to postmortem histological analyses that require the killing of large numbers of animals to validate quantitative findings, thus increasing research costs and decreasing clinical relevance of therapies targeted at promoting or hindering the process of angiogenesis. Further development of molecular imaging approaches should facilitate the translation of novel therapeutics into clinical trials and improve patient management by permitting non-invasive assessment of serial angiogenic responses to treatment.

\section{Angiogenesis}

Angiogenesis, in general, is the physiological process by which new capillaries are formed from pre-existing vessels. Formation of new microvascular networks can be of critical significance in the setting of cardiovascular disease, as angiogenesis can assist in restoring blood flow and perfusion to skeletal muscle or cardiac tissue exposed to hypoxic conditions and potentially prevent the onset of tissue necrosis. Angiogenesis is regulated through multiple angiogenic factors, cells, and the extracellular matrix (ECM), and can be stimulated in the setting of various conditions that may be associated with cardiovascular disease, such as ischemia, hypoxia, inflammation, and alterations in vascular shear stress [5]. The process of angiogenesis can be described by several stages. These stages are first characterized by the initial activation of endothelial cells that leads to increased permeability and proliferation of endothelial cells. Next, the extracellular matrix (ECM) is degraded, with the assistance of multiple factors such as matrix metalloproteinases (MMPs) and integrins, which facilitates infiltration of endothelial cells. Following ECM breakdown and endothelial cell migration, the development and eventual stabilization of a new capillary occurs [6].

Numerous factors have been attributed to stimulating angiogenesis in the setting of tissue hypoxia, such as hypoxia-inducible factor 1 (HIF-1), vascular endothelial growth factor (VEGF), platelet-derived growth factor (PDGF), fibroblast growth factor-2 (FGF-2), transforming growth factor beta (TGF- $\beta$ ), and angiopoietins [7-10]. With the assistance of the aforementioned angiogenic mediators, additional signaling events result in participation of endothelial cells, vascular smooth muscle cells, blood-derived macrophages, and circulating stem cells in the angiogenic process [11]. In addition to macrophages, a variety of other inflammatory cells and mediators can play important roles in angiogenesis. Through hypoxia-induced stimulation, inflammatory cells can release multiple angiogenesis-stimulating factors and proteases, such as vascular endothelial growth factor (VEGF), tumor necrosis factor- $\alpha$ (TNF- $\alpha)$, cytokines, and MMPs. Inflammatory cells can also release reactive oxygen species (ROS), which can serve as stimulators of the angiogenic process [6].

While there are many known mediators of the angiogenic process, VEGF has been established as one of the most influential contributors to vascular development. Five VEGF-related growth factors exist, which stimulate angiogenesis through binding to three tyrosine kinase receptors (VEGFR-1, VEGR-2, and VEGR-3) [12]. Along with VEGF's established role, integrins are also considered to be important regulators of the angiogenic process. These transmembrane receptors are capable of mediating cell-tocell and cell-to-ECM interactions and can modulate cell adhesion, migration, proliferation, differentiation, and survival. The $\alpha \mathrm{v} \beta 3$ integrin has been of particular interest due to significant involvement in the regulation of cell interactions with the ECM and endothelial cell migration [13]. As the field of molecular imaging continues to develop, VEGF and the $\alpha v \beta 3$ integrin have emerged as the primary targets for non-invasive evaluation of cardiovascular-related angiogenesis; however, multiple targets are currently available for the evaluation of angiogenesis in the setting of cancer, cardiovascular disease, and inflammation. Established targets for molecular imaging of angiogenesis have been grouped into three categories: 1) non-endothelial cell targets (e.g., monocytes, macrophages, and stem cells), endothelial cell targets (e.g., VEGF, receptors, and integrins), and 3) ECM proteins and proteases [5]. Future discussion will be limited to translational cardiovascular imaging approaches that have been performed for the noninvasive assessment of myocardial and peripheral angiogenesis.

\section{Radiotracer imaging of myocardial angiogenesis}

Angiogenesis is enhanced in the setting of myocardial ischemia as a physiological response to ischemic insult, particularly in the setting of myocardial infarction (MI). In 
animal models of MI, angiogenic activation after MI can be confirmed by histopathologic analysis, such as capillary density measurement. However, non-invasive imaging is currently the only practical method for evaluating angiogenesis in vivo. Many studies have reported augmented angiogenesis following MI as well as cerebral infarction by using radiotracer imaging methods [14, 15]. Among radiotracer-based studies targeted at the angiogenic process, the RGD peptide (composed of L-arginine, glycine, and Laspartic acid) moiety targeted at the integrin $\alpha v \beta 3$ has been most widely applied. The $\alpha v \beta 3$ integrin is expressed on the surface of the endothelium that is activated for angiogenesis. Additionally, the expression of $\alpha v \beta 3$ integrin is present on activated macrophages. Thus, the RGD peptide moiety targets for both post-infarct angiogenesis and macrophage accumulation. The uptake of RGD peptide agents in the post-infarct myocardium has been demonstrated to be closely related to expression of CD31 (angiogenesis marker) and CD68 (macrophage marker), as well as integrin expression [16-20].

Many studies have reported visualization and quantification of increased angiogenesis following MI using radiotracer-based imaging approaches. Early application of radiotracer-based imaging for the evaluation of post-MI angiogenesis was performed using scintigraphy or single photon emission computed tomography (SPECT). SPECT imaging with indium-111 ( ${ }^{111}$ In)-RP748 in rat and canine models of MI have demonstrated a 2- to 4-fold increase in myocardial uptake within infarcted myocardium compared to remote regions $[16,21,22]$. Technetium-99 $\mathrm{m}\left({ }^{99 \mathrm{~m}} \mathrm{Tc}\right)$ is currently a more readily accessible radioisotope than ${ }^{111} \mathrm{In}$, and studies using ${ }^{99 \mathrm{~m}}$ Tc-labeled RGD peptides $\left({ }^{99 \mathrm{~m}} \mathrm{Tc}\right.$ RAFT-RGD and ${ }^{99 m}$ Tc-NC100692) have shown increased accumulation of these radiotracers within infarcted myocardium and the border zone of infarction (Fig. 1) [17, 23]. PET imaging has become more widely available for pre-clinical and clinical imaging, and angiogenesis-targeted radiotracers for PET have been investigated more extensively than radiotracers for gamma cameras. Flourine$18\left({ }^{18} \mathrm{~F}\right)$-galacto-RGD [24, 25], copper-64 $\left({ }^{64} \mathrm{Cu}\right)-\mathrm{VEGF}$ [26], ${ }^{18}$ F-PRGD [18], ${ }^{64} \mathrm{Cu}-\mathrm{TRC} 105$ [27], and several gallium-68 ( ${ }^{68} \mathrm{Ga}$ )-labeled RGD peptide agents [19, 20, 28, 29] have been reported to be effective for PET imaging of post-infarct angiogenesis (Table 1). Pre-clinical PET studies in animal models of MI have demonstrated a 1.5- to 3.9-fold increase in angiogenesis activity from baseline levels within infarcted myocardium [18-20, 27]. In addition to pre-clinical application, some PET angiogenesistargeted tracers have reached the clinical setting for the assessment of post-MI remodeling and have demonstrated approximately a 2-fold increase in radiotracer uptake within infarcted myocardium when compared to normal or remote myocardium $[25,28]$.

The natural time course of post-infarct remodeling can be serially evaluated using non-invasive angiogenesis-targeted imaging. Despite differences that exist in the animal models studied and the radiotracers incorporated, increased angiogenesis (as assessed by $\alpha v \beta 3$ integrin activation) has commonly been observed within 1-3 days after occurrence of MI [16, 22, 24, 25]. In the majority of studies, myocardial uptake of radiotracers reached peak levels at 1-3 weeks following infarction [18, 23, 24, 28]. However, considerable variation in the persistence of angiogenesis signal has been demonstrated, with some studies reporting that radiotracer uptake is normalized as early as 17 days following infarction [27], whereas other studies report
Fig. 1 SPECT imaging of myocardial angiogenesis in a canine model of myocardial infarction obtained 2 weeks following $6 \mathrm{~h}$ balloon occlusion of the left anterior descending artery. Focal uptake of ${ }^{99 \mathrm{~m}} \mathrm{Tc}$ $\mathrm{NC} 100692$ is observed in the region of myocardial infarction (denoted by white arrow), which is localized to a region of decreased perfusion, as identified by ${ }^{201} \mathrm{Tl}$ SPECT (yellow arrow) (color figure online)

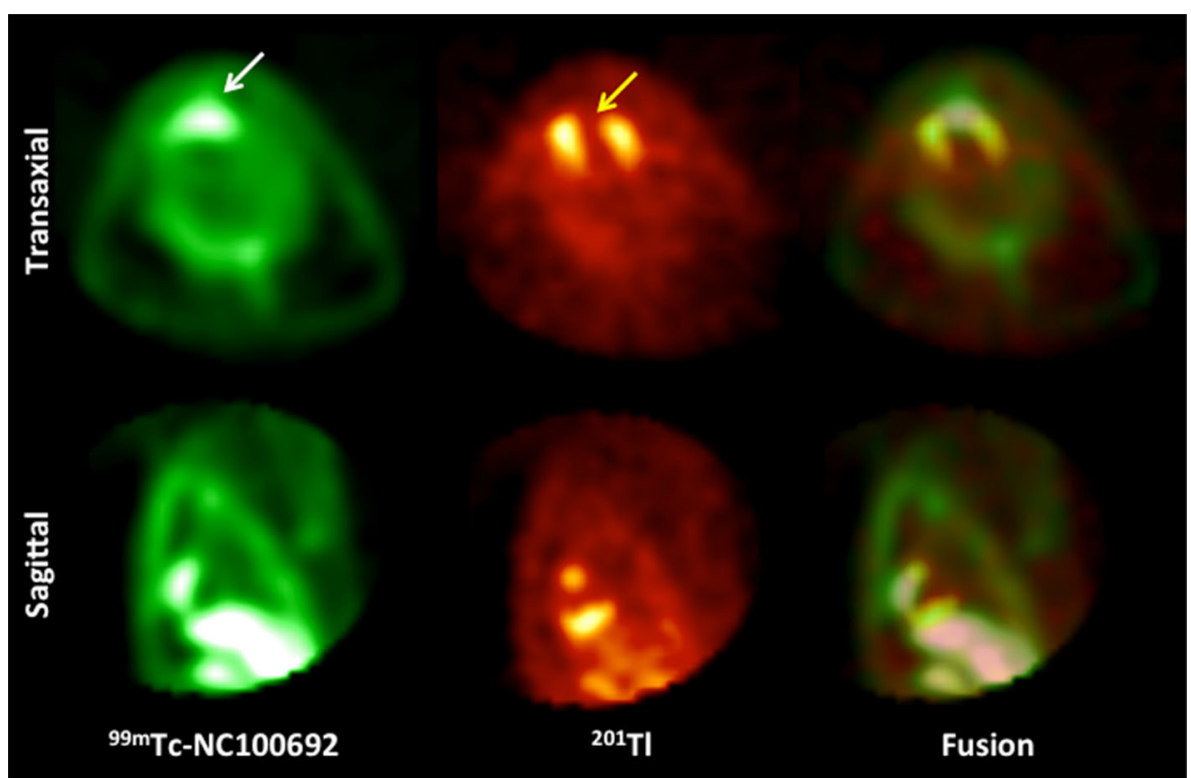


Table 1 Radiotracers for imaging of cardiovascular angiogenesis

\begin{tabular}{|c|c|c|}
\hline Biologic target & Radiotracer & Modality \\
\hline \multicolumn{3}{|l|}{ Myocardial } \\
\hline \multirow[t]{12}{*}{ Pre-clinical } & ${ }^{64} \mathrm{Cu}-\mathrm{DOTA}-\mathrm{VEGF}_{121}[26]$ & PET \\
\hline & ${ }^{64} \mathrm{Cu}-\mathrm{NOTA}-\mathrm{TRC} 105$ [27] & PET \\
\hline & ${ }^{18}$ F-Galacto-RGD [24, 29] & PET \\
\hline & ${ }^{68}$ Ga-NOTA-RGD $[19,20]$ & PET \\
\hline & ${ }^{68}$ Ga-PRGD2 [28] & PET \\
\hline & ${ }^{68}$ Ga-NODAGA-RGD [29] & PET \\
\hline & ${ }^{68} \mathrm{Ga}-\mathrm{TRAP}(\mathrm{RGD})_{3}[29]$ & PET \\
\hline & ${ }^{18}$ F-AIF-NOTA-PRGD2 [18] & PET \\
\hline & ${ }^{123}$ I-Gluco-RGD [30] & SPECT \\
\hline & ${ }^{99 \mathrm{~m}} \mathrm{Tc}-\mathrm{NC} 100692[31,44,45]$ & SPECT \\
\hline & ${ }^{99 m}$ Tc-RAFT-RGD [17] & SPECT \\
\hline & ${ }^{111}$ In-RP748 [16, 21, 22] & SPECT \\
\hline \multirow[t]{2}{*}{ Clinical trials } & ${ }^{18}$ F-Galacto-RGD [25] & PET \\
\hline & ${ }^{99 m}$ Tc-NC100692 [33] & SPECT \\
\hline \multicolumn{3}{|l|}{ Peripheral } \\
\hline & ${ }^{76} \mathrm{Br}$-nanoprobe [35] & PET \\
\hline & ${ }^{68}$ Ga-NOTA-RGD [41] & PET \\
\hline & ${ }^{64} \mathrm{Cu}$-DOTA-CANF-comb [43] & PET \\
\hline & ${ }^{64} \mathrm{Cu}-\mathrm{DOTA}-\mathrm{VEGF}_{121}[42]$ & PET \\
\hline & ${ }^{64} \mathrm{Cu}$-NOTA-TRC105 [34, 46] & PET \\
\hline & ${ }^{99 m}$ Tc-NC100692 [36, 40, 47, 48] & SPECT \\
\hline & ${ }^{111}{ }^{1 n}-V_{E G F}{ }_{121}[37]$ & SPECT \\
\hline & ${ }^{125} \mathrm{I}-\mathrm{c}(\mathrm{RGD}(\mathrm{I}) \mathrm{yV})$ [39] & SPECT \\
\hline
\end{tabular}

persistent uptake for up to $4-6$ months $[18,24]$. Work in our own laboratory has demonstrated normalization of the angiogenic response at approximately 6-9 weeks following MI in a canine model of temporary left anterior descending artery (LAD) occlusion [23]. The differences in the time courses for angiogenesis that have been observed following MI may be related to a number of variables, including the animal model being utilized and the duration and site of coronary occlusion.

In recent years, therapeutic angiogenesis using various growth factors and gene- or cell-based therapies have been attempted to induce angiogenesis and relieve myocardial ischemia. Pre-clinical application of SPECT and PET imaging has demonstrated that radiotracer-based imaging of angiogenesis may be useful for monitoring the effect of therapeutic treatments. In a swine model of hibernating myocardium, focal uptake of iodine-123 $\left({ }^{123} \mathrm{I}\right)$-gluco-RGD was found to correspond with sites of prior intra-myocardial VEFG injections and correlated with postmortem analysis of capillary density [30]. In addition to the use of ${ }^{123}$ I-gluco-RGD for monitoring of VEGF therapy in infarcted myocardium, ${ }^{99 \mathrm{~m}} \mathrm{Tc}-\mathrm{RAFT}$-RGD SPECT and ${ }^{68} \mathrm{Ga}-$ NOTA-RGD PET imaging have also been used to evaluate VEGF and fibroblast growth factor therapies in rat models of MI [19, 30]. In these studies, radiotracer uptake was observed in accordance with increased capillary density and other markers of angiogenesis. Other pre-clinical work using micro-SPECT/CT imaging of ${ }^{99 \mathrm{~m}} \mathrm{Tc}-\mathrm{NC} 100692$ has demonstrated potential for evaluating the angiogenic response to insulin-like growth factor-1 gene therapy in a rat model of MI, suggesting that monitoring of therapeutic efficacy with angiogenesis imaging could have potential for clinical application [31].

Molecular imaging of myocardial angiogenesis has been translated to the clinical setting for the evaluation of patients with hypertrophic cardiomyopathy [32] and heart failure [33], as well as following acute MI [25]. In the evaluation of acute MI, focal uptake of ${ }^{18} \mathrm{~F}$-galacto-RGD has been shown to localize to the MI area defined by delayed enhancement magnetic resonance (MR) imaging [25]. The use of ${ }^{99 \mathrm{~m}} \mathrm{Tc}-\mathrm{NC} 100692$ in patients with hypertrophic cardiomyopathy has also demonstrated highergrade radiotracer uptake within regions of fibrosis detected by MR [32]. Additionally, ${ }^{99 \mathrm{~m}} \mathrm{Tc}-\mathrm{NC} 100692$ has been used to evaluate the therapeutic efficacy of bone marrowderived stem cell therapy in patients with heart failure; however, radiotracer uptake was found to be only mildly correlated with functional improvement [33]. Further investigation into the long-term prognostic role of monitoring the angiogenesis process in the setting of hypertrophic heart disease, following MI, or in the evaluation of therapeutic treatments is warranted, while the context of clinical conditions should also be considered in the interpretation of molecular imaging strategies.

\section{Radiotracer imaging of peripheral angiogenesis}

In addition to being a useful tool for non-invasive assessment of myocardial ischemia, molecular imaging of angiogenesis has also proven to be effective in the evaluation of limb ischemia. Ischemia-induced angiogenesis has been demonstrated to occur in various pre-clinical models of femoral artery occlusion, such as rodent [34-36], rabbit [37], and pig models [38]. Although molecular imaging of peripheral angiogenesis has not yet reached clinical application, pre-clinical work has shown promising results for non-invasive serial analysis of the angiogenic process, with the primary vascular targets of choice being VEGF receptors and the $\alpha v \beta 3$ integrin.

In one of the first molecular imaging studies targeting peripheral angiogenesis, Lu et al. [37] targeted VEGF receptors in a rabbit model of unilateral hind limb ischemia using planar imaging and gamma counting of ${ }^{111} \mathrm{In}$-labeled recombinant human $\mathrm{VEGF}_{121}$. Ten days following surgically induced limb ischemia, both imaging and gamma counting demonstrated increased uptake of ${ }^{111}$ In-labeled 
$\mathrm{VEGF}_{121}$ in ischemic vs. control hind limb tissue. Furthermore, immunohistochemical analysis of ischemic tissue confirmed an increase in VEGF receptor expression.

Along with targeting of VEGF receptors, early work in mouse models of hind limb ischemia also targeted the $\alpha \mathrm{v} \beta 3$ integrin using iodine-125 ( $\left.{ }^{125} \mathrm{I}\right)$ - and ${ }^{99 \mathrm{~m}}$ Tc-labeled RGD peptides [39, 40]. Lee et al. [39] evaluated ${ }^{125} \mathrm{I}-$ c(RGD(I)yV uptake in ischemic hind limb tissue using gamma counting and found significantly increased radiotracer uptake at days 3, 8, and 14 following femoral ablation, which was also associated with gradual recovery of limb perfusion (evaluated by laser Doppler). Additionally, radiotracer uptake was validated through immunohistochemistry that demonstrated a pronounced increase in $\alpha v \beta 3$ integrin expression in ischemic tissue. Further evaluation of serial changes in $\alpha v \beta 3$ integrin expression during the angiogenic process has been performed in the ischemic hind limb through non-invasive in vivo planar imaging of ${ }^{99 m}$ Tc-NC100692 [40]. Imaging demonstrated radiotracer uptake in the distal hind limb that increased at 3 days and peaked at 7 days following femoral artery occlusion. Image quantification was validated by gamma counting of hind limb tissue, and increased radiotracer uptake corresponded with serial increases in capillary density. Additionally, immunofluorescent staining confirmed specificity and co-localization of NC100692 (targeting the $\alpha v \beta 3$ integrin) to endothelial cells. Further work has validated and applied a semiautomated quantitative approach for serial micro-SPECT/CT imaging of ${ }^{99 \mathrm{~m}} \mathrm{Tc}$ NC100692 and demonstrated high reproducibility and accuracy of this approach for evaluating angiogenesis within specific volumes of interest in the hind limbs of wild-type and endothelial nitric oxide synthase (eNOS) knockout mice [36]. Peak ${ }^{99 m}$ Tc-NC100692 uptake was found to occur 1 week following femoral artery occlusion in both wild-type and eNOS knockout mice; however, there was a significant decrease in radiotracer uptake in eNOS-deficient mice relative to wild-type mice at the 1 week postocclusion time point. These results suggest that SPECT/CT imaging of angiogenesis may be a useful non-invasive tool for future translational studies evaluating different stages and forms of disease, such as peripheral vascular disease and diabetes. Work from our laboratory has recently focused on translating ${ }^{99 \mathrm{~m}}$ Tc-NC100692 SPECT/CT imaging from a small to large animal model of hind limb ischemia to non-invasively evaluate serial changes in angiogenesis and has demonstrated focal radiotracer uptake within ischemic tissue and surgical site (Fig. 2).

In addition to ${ }^{111}$ In and ${ }^{99 \mathrm{~m}} \mathrm{Tc}$ labeling of angiogenesistargeted compounds, ${ }^{68} \mathrm{Ga}$ [41] and ${ }^{64} \mathrm{Cu}$ [42] have also been used for non-invasive assessment of angiogenesis in the mouse model of hind limb ischemia. In a feasibility study by Jeong et al. [41], ${ }^{68}$ Ga-NOTA-RGD targeted at the $\alpha v \beta 3$ integrin demonstrated specific uptake by angiogenic hind limb tissue, high stability, high affinity for the $\alpha v \beta 3$ integrin, and good pharmacokinetic properties. In another study utilizing micro-PET imaging of ${ }^{64} \mathrm{Cu}$ $\mathrm{VEGF}_{121}$, radiotracer uptake was significantly increased in ischemic hind limb tissue 8 days following femoral artery ligation and subsequently displayed a steady decrease in uptake over the following 3 weeks [42]. Interestingly, ${ }^{64} \mathrm{Cu}-\mathrm{VEGF}_{121}$ uptake was also significantly higher in the skeletal muscle of mice exposed to hind limb ischemia and exercise training when compared to non-exercised controls, with radiotracer uptake being strongly correlated with VEGFR2 tissue levels.

${ }^{64} \mathrm{Cu}$ has also been used for labeling of C-type atrial natriuretic factor (CANF)-conjugated comblike nanoprobes for detecting the upregulation of natriuretic peptide clearance receptor (NPR-C) in the setting of hind limb ischemia [43]. PET imaging of targeted ${ }^{64} \mathrm{Cu}$-DOTA-CANF-comb demonstrated a nearly 6-fold increase in radiotracer uptake at $24 \mathrm{~h}$ post-femoral artery ligation in the ischemic hind limb relative to the non-ischemic control limb, which corresponded with gradual recovery of blood flow, indicative of a progressive angiogenic response. Immunohistochemistry established co-localization of NPR-C in endothelial and smooth muscle cells, and specificity of
Fig. 2 Serial imaging of angiogenesis in a pig model of hind limb ischemia. Fused ${ }^{99 \mathrm{~m}} \mathrm{Tc}-\mathrm{NC} 100692$ SPECT and CT axial images at 2 (a) and 4 (b) weeks post-femoral artery occlusion demonstrate focal uptake of ${ }^{99 \mathrm{~m}} \mathrm{Tc}-\mathrm{NC} 100692$ in the lateral aspect of the hind limb (white arrows), as well as in the surgical site (yellow arrows) (color figure online)
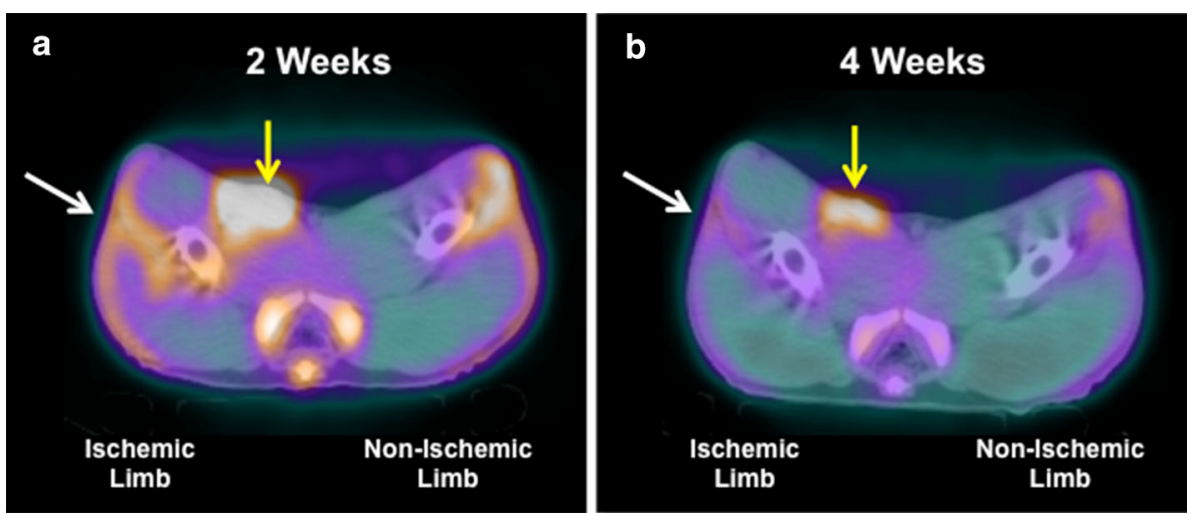
${ }^{64} \mathrm{Cu}$-DOTA-CANF-comb to NPR-C was demonstrated via competitive receptor blocking. In addition to ${ }^{64} \mathrm{Cu}$-labeled nanoprobes, Almutairi et al. developed biodegradable dendritic nanoprobes that were functionalized with heterobifunctional polyethylene oxide (PEO) chains and used for targeted PET imaging of the $\alpha v \beta 3$ integrin in hind limb angiogenesis [35]. PEO chains were fitted with RGD motifs for selective targeting of the $\alpha v \beta 3$ integrin. Labeling of nanoprobes with ${ }^{125}$ I for cell-based assays demonstrated a 6-fold increase in nanoprobe uptake within $\alpha v \beta 3$-positive cells. Additionally, in vivo PET imaging of bromine-76 $\left({ }^{76} \mathrm{Br}\right)$-labeled nanoprobes confirmed specific uptake within angiogenic hind limb tissue.

Most recently, ${ }^{64} \mathrm{Cu}$-labeleling of NOTA-conjugated TRC105, a CD105 antibody, has been developed for PET imaging of angiogenesis in the mouse model of hind limb ischemia [27]. The first serial imaging study evaluating ${ }^{64} \mathrm{Cu}-$ TRC105 demonstrated peak radiotracer uptake at 3 and 10 days following femoral artery ligation, which was validated using histology and reverse transcription polymerase chain reaction (RT-PCR) of hind limb tissue. Further application of ${ }^{64} \mathrm{Cu}$-TRC105 has demonstrated the ability of this radiotracer to non-invasively track serial changes in angiogenesis following pravastatin therapy in the setting of hind limb ischemia and has showed significantly higher levels of radiotracer uptake in treatment vs. non-treatment groups [34]. These findings suggest that non-invasive molecular imaging of peripheral angiogenesis may have potential for evaluating and monitoring serial responses to clinical treatment in patients with peripheral vascular disease.

\section{Conclusions}

Future translation of angiogenesis-targeted imaging with radiotracer-based approaches may advance evaluation of myocardial ischemia/infarction and peripheral vascular disease in the clinical setting. Additionally, molecular imaging of angiogenesis may allow for improved non-invasive assessment of serial responses to medical treatment, including revascularization, exercise training, and novel therapeutics. Ongoing SPECT and PET imaging studies targeted at the angiogenic process should focus on the development of radiotracers that will result in reasonable levels of ionizing radiation exposure for patients while also possessing high affinity for angiogenesis-related vascular targets, thereby facilitating clinical translational of molecular imaging approaches that should allow for integration of physiologic information with standard anatomic and clinical indices.

Acknowledgments This work was supported in part by NIH grants T32 HL098069 and R01 HL65662 to Dr. Sinusas, and American Heart Association award 14CRP20480404 to Dr. Stacy.
Conflict of interest Dr. Sinusas receives research support and NC100692 from GE Healthcare. Drs. Stacy and Paeng have no conflicts of interest to disclose.

\section{References}

1. Stacy MR, Sinusas AJ. Emerging imaging modalities in regenerative medicine. Curr Pathobiol Rep. 2015;3:27-36.

2. Stacy MR, Zhou W, Sinusas AJ. Radiotracer imaging of peripheral vascular disease. J Nucl Med. 2013;54:2104-10.

3. Cal-Gonzalez J, Lage E, Herranz E, Vicente E, Udias JM, Moore $\mathrm{SC}$, et al. Simulation of triple coincidences in PET. Phys Med Biol. 2015;60:117-36.

4. Andreyev A, Celler A. Dual-isotope PET using positron-gamma emitters. Phys Med Biol. 2011;56:4539-56.

5. Stacy MR, Maxfield MW, Sinusas AJ. Targeted molecular imaging of angiogenesis in PET and SPECT: a review. Yale J Biol Med. 2012;85:75-86.

6. Hong H, Chen F, Zhang Y, Cai W. New radiotracers for imaging of vascular targets in angiogenesis-related diseases. Adv Drug Deliv Rev. 2014;76:2-20.

7. Shweiki D, Itin A, Soffer D, Keshet E. Vascular endothelial growth factor induced by hypoxia may mediate hypoxia-initiated angiogenesis. Nature. 1992;359:843-5.

8. Brogi E, Schatteman G, Wu T, Kim EA, Varticovski L, Keyt B, et al. Hypoxia-induced paracrine regulation of vascular endothelial growth factor receptor expression. J Clin Invest. 1996;97:469-76.

9. Banai S, Jaklitsch MT, Shou M, Lazarous DF, Scheinowitz M, Biro S, et al. Angiogenic-induced enhancement of collateral blood flow to ischemic myocardium by vascular endothelial growth factor in dogs. Circulation. 1994;89:2183-9.

10. Li J, Brown LF, Hibberd MG, Grossman JD, Morgan JP, Simons M. VEGF, flk-1, and flt-1 expression in a rat myocardial infarction model of angiogenesis. Am J Physiol. 1996;270:H1803-11.

11. Dufraine J, Funahashi Y, Kitajewski J. Notch signaling regulates tumor angiogenesis by diverse mechanisms. Oncogene. 2008;27:5132-7.

12. Eichmann A, Simons M. VEFG signaling inside vascular endothelial cells and beyond. Curr Opin Cell Biol. 2012;24:188-93.

13. Schwartz MA, Schaller MD, Ginsberg MH. Integrins: emerging paradigms of signal transduction. Ann Rev Cell Dev Biol. 1995;11:549-99.

14. Cai W, Guzman R, Hsu AR, Wang H, Chen K, Sun G, et al. Positron emission tomography imaging of poststroke angiogenesis. Stroke. 2009;40:270-7.

15. Choi H, Phi JH, Paeng JC, Kim SK, Lee YS, Jeong JM, et al. Imaging of integrin $\mathrm{a}(\mathrm{V}) \mathrm{B}(3)$ expression using (68)Ga-RGD positron emission tomography in pediatric cerebral infarct. Mol Imaging. 2013;12:213-7.

16. Kalinowski L, Dobrucki LW, Meoli DF, Dione DP, Sadeghi MM, Madri JA, et al. Targeted imaging of hypoxia-induced integrin activation in myocardium early after infarction. J Appl Physiol. 2008;104:1504-12.

17. Dimastromatteo J, Riou LM, Ahmadi M, Pons G, Pellegrini E, Broisat A, et al. In vivo molecular imaging of myocardial angiogenesis using the alpha(v)beta3 integrin-targeted tracer 99mTc-RAFT-RGD. J Nucl Cardiol. 2010;17:435-43.

18. Gao H, Lang L, Guo N, Cao F, Quan Q, Hu S, et al. PET imaging of angiogenesis after myocardial infarction/reperfusion using a one-step labeled integrin-targeted tracer 18F-AIF-NOTAPRGD2. Eur J Nucl Med Mol Imaging. 2012;39:683-92.

19. Eo JS, Paeng JC, Lee S, Lee YS, Jeong JM, Kang KW, et al. Angiogenesis imaging in myocardial infarction using $68 \mathrm{Ga}$ - 
NOTA-RGD PET: characterization and application to therapeutic efficacy monitoring in rats. Coron Artery Dis. 2013;24:303-11.

20. Menichetti L, Kusmic C, Panetta D, Arosio D, Petroni D, Matteucci M, et al. MicroPET/CT imaging of alpha(v)beta(3) integrin via a novel (68)Ga-NOTA-RGD peptidomimetic conjugate in rat myocardial infarction. Eur $\mathrm{J}$ Nucl Med Mol Imaging. 2013;40:1265-74.

21. Meoli DF, Sadeghi MM, Krassilnikova S, Bourke BN, Giordano FJ, Dione DP, et al. Noninvasive imaging of myocardial angiogenesis following experimental myocardial infarction. J Clin Invest. 2004;113:1684-91.

22. Dobrucki LW, Meoli DF, Hu J, Sadeghi MM, Sinusas AJ. Regional hypoxia correlates with the uptake of a radiolabeled targeted marker of angiogenesis in rat model of myocardial hypertrophy and ischemic injury. $J$ Physiol Pharmacol. 2009;60:117-23.

23. Paeng JC, Bregasi A, Sahul Z, Kalinowski L, Dobrucki LW, Brennan M, et al. Serial reference tissue-based quantitative and volumetric analysis of integrin-targeted angiogenesis imaging: chronic canine model of myocardial infarction. J Nucl Med. 2014;55:1710.

24. Higuchi T, Bengel FM, Seidl S, Watzlowik P, Kessler H, Hegenloh $\mathrm{R}$, et al. Assessment of alphavbeta3 integrin expression after myocardial infarction by positron emission tomography. Cardiovasc Res. 2008;78:395-403.

25. Makowski MR, Ebersberger U, Nekolla S, Schwaiger M. In vivo molecular imaging of angiogenesis, targeting alphavbeta3 integrin expression, in a patient after acute myocardial infarction. Eur Hear. J. 2008;29:2201.

26. Rodriguez-Porcel M, Cai W, Gheysens O, Willmann JK, Chen K, Wang $\mathrm{H}$, et al. Imaging of VEGF receptor in a rat myocardial infarction model using PET. J Nucl Med. 2008;49:667-73.

27. Orbay H, Zhang Y, Valdovinos HF, Song G, Hernandez R, Theuer CP, et al. Positron emission tomography imaging of CD105 expression in a rat myocardial infarction model with (64)Cu-NOTA-TRC105. Am J Nucl Med Mol Imaging. 2013;4:1-9.

28. Sun Y, Zeng Y, Zhu Y, Feng F, Xu W, Wu C, et al. Application of (68)Ga-PRGD2 PET/CT for alpha(v)beta(3)-integrin imaging of myocardial infarction and stroke. Theranostics. 2014:4:778-86.

29. Laitinen I, Notni J, Pohle K, Rudelius M, Farrell E, Nekolla SG, et al. Comparison of cyclic RGD peptides for alpha(v)beta(3) integrin detection in a rat model of myocardial infarction. EJNMMI Res. 2013;3:38.

30. Johnson LL, Schofield L, Donahay T, Bouchard M, Poppas A, Haubner R. Radiolabeled arginine-glycine-aspartic acid peptides to image angiogenesis in swine model of hibernating myocardium. JACC Cardiovasc Imaging. 2008;1:500-10.

31. Dobrucki L, Tsutsumi Y, Kalinowski L, Dean J, Gavin M, Sen S, et al. Analysis of angiogenesis induced by local IGF-1 expression after myocardial infarction using microSPECT-CT imaging. J Mol Cell Cardiol. 2010;48:1071-9.

32. Cocker MS, Dwivedi G, Marvin B, Poirier M, Dennie C, Wells $\mathrm{G}$, et al. Integrin imaging for the detection of diffuse myocardial fibrosis in patients with hypertrophic cardiomyopathy: direct comparison between single-photon emission computed tomography and cardiovascular magnetic resonance. The SCAR study. Am Hear Assoc Sci Sess. 2012;A19661.

33. Mozid AM, Holstensson M, Choudhury T, Ben-Haim S, Allie R, Martin J, et al. Clinical feasibility study to detect angiogenesis following bone marrow stem cell transplantation in chronic ischaemic heart failure. Nucl Med Commun. 2014;35:839-48.

34. Orbay H, Hong H, Koch JM, Valdovinos HF, Hacker TA, Theuer $\mathrm{CP}$, et al. Pravastatin stimulates angiogenesis in a murine hindlimb ischemia model: a positron emission tomography imaging study with (64)Cu-NOTA-TRC105. Am J Transl Res. 2013;6:54-63.

35. Almutairi A, Rossin R, Shokeen M, Hagooly A, Ananth A, Capoccia B, et al. Biodegradable dendritic positron-emitting nanoprobes for the noninvasive imaging of angiogenesis. Proc Natl Acad Sci USA. 2009;106:685-90.

36. Dobrucki LW, Dione DP, Kalinowski L, Dione D, Mendizabal $\mathrm{M}, \mathrm{Yu}$ J, et al. Serial noninvasive targeted imaging of peripheral angiogenesis: validation and application of a semiautomated quantitative approach. J Nucl Med. 2009;50:1356-63.

37. Lu E, Wagner WR, Schellenberger U, Abraham JA, Klibanov $\mathrm{AL}$, Woulfe SR, et al. Targeted in vivo labeling of receptors for vascular endothelial growth factor: approach to identification of ischemic tissue. Circulation. 2003;108:97-103.

38. Stacy MR, Yu DY, Maxfield MW, Jaba IM, Jozwik BP, Zhuang $\mathrm{ZW}$, et al. Multimodality imaging approach for serial assessment of regional changes in lower extremity arteriogenesis and tissue perfusion in a porcine model of peripheral arterial disease. Circ Cardiovasc Imaging. 2014;7:92-9.

39. Lee K, Jung K, Song S, Kim DH, Lee BC, Sung HJ, et al. Radiolabeled RGD uptake and alpha(v) integrin expression is enhanced in ischemic murine hindlimbs. $J$ Nucl Med. 2005;46:472-8.

40. Hua J, Dobrucki LW, Sadeghi MM, Zhang J, Bourke BN, Cavaliere $\mathrm{P}$, et al. Noninvasive imaging of angiogenesis with a $(99 \mathrm{~m}) \mathrm{Tc}$-labeled peptide targeted at alpha(v)beta(3) integrin after murine hindlimb ischemia. Circulation. 2005;111:3255-60.

41. Jeong JM, Hong MK, Chang YS, Lee Y-S, Kim YJ, Cheon GJ, et al. Preparation of a promising anigogenesis PET imaging agent: (68)Ga-labeled c(RGDyK)-isothiocyanatobenzyl1-1,4,7triazacyclononane-1,4,7-triacetic acid and feasibility studies in mice. J Nucl Med. 2008;49:830-6.

42. Willmann JK, Chen K, Wang H, Paulmurugan R, Rollins M, Cai $\mathrm{W}$, et al. Monitoring of the biological response to murine hindlimb ischemia with $64 \mathrm{Cu}$-labeled vascular endothelial growth factor-121 positron emission tomography. Circulation. 2008;117:915-22.

43. Liu Y, Pressly ED, Abendschein DR, Hawker CJ, Woodard GE, Woodard PK, et al. Targeting angiogenesis using a C-type atrial natriuretic factor-conjugated nanoprobe and PET. J Nucl Med. 2011;52:1956-63.

44. Li S, Sinusas AJ, Dobrucki LW, Liu YH. New approach to quantification of molecularly targeted radiotracer uptake from hybrid cardiac SPECT/CT: methodology and validation. J Nucl Med. 2013;54:2175-81.

45. Lindsey ML, Escobar GP, Dobrucki LW, Goshorn DK, Bouges S, Mingoia JT, et al. Matrix metalloproteinase-9 gene deletion facilitates angiogenesis after myocardial infarction. Am J Physiol Hear Circ Physiol. 2006;290:H232-9.

46. Orbay H, Zhang Y, Hong H, Hacker TA, Valdovinos HF, Zagzebski JA, et al. Positron emission tomography imaging of angiogenesis in a murine hindlimb ischemia model with (64)Culabeled TRC105. Mol Pharm. 2013;10:2749-56.

47. Mehra VC, Jackson E, Zhang XM, Jiang XC, Dobrucki LW, Yu $\mathrm{J}$, et al. Ceramide-activated phosphatase mediates fatty acid-induced endothelial VEGF resistance and impaired angiogenesis. Am J Pathol. 2014;184:1562-76.

48. Hedhli N, Dobrucki LW, Kalinowski A, Zhuang ZW, Wu X, Russell RR 3rd, et al. Endothelial-derived neuregulin is an important mediator of ischaemia-induced angiogenesis and arteriogenesis. Cardiovasc Res. 2012;93:516-24. 